\title{
HOBNAIL HEMANGIOMA: A IMMUNOHISTOCHEMICAL STUDY AND LITERATURE REVIEW
}

\author{
Maria Hejnold ${ }^{1}$, Grzegorz Dyduch ${ }^{1}$, Izabela Mojsa ${ }^{2}$, Krzysztof Okoń ${ }^{1}$ \\ 1Department of Pathomorphology, Collegium Medicum, Jagiellonian University, Kraków, Poland \\ 2Department of Oral Surgery, Collegium Medicum, Jagiellonian University, Kraków, Poland
}

\begin{abstract}
Hobnail hemangioma is a rare vascular lesion with unusual morphology, including bland cells with hobnail appearance, a biphasic grow pattern with superficial dilated vessels and slit-like vessels in a deeper portion of the lesion and the infiltrative pattern of later growth, which may cause misdiagnosis. The differential diagnosis with hemangioendothelioma variants, low grade angiosarcomas and Kaposi sarcoma is of particular concern. The lack of recognition of this uncommon entity may result in excessive and unnecessary treatment. Immunohistochemistry stains play an important role in hobnail hemangioma differential diagnosis. Here, we present the results of our researches and also review of the literature as well as discussion about usage of immunohistochemistry in the diagnosis of $\mathrm{HH}$.
\end{abstract}

Key words: hemangioma, hobnail cells, immunohistochemistry.

\section{Introduction}

Hobnail hemangioma $(\mathrm{HH})$ was first described as targetoid hemosiderotic hemangioma by Santa Cruz et al. in 1988 [1]. Although entirely benign, its peculiar morphology requires careful differential diagnosis with low grade vascular cancers. The nature of $\mathrm{HH}$ is somehow controversial. The aim of the present study was to investigate the expression of a number of markers that could clarify the origin of $\mathrm{HH}$. We have recently described one such lesion [2] and now we present the results of immunohistochemistry within a short series of HHs. Our group previously performed similar studies on the origin of conventional hemangiomas $[3,4]$.

\section{Material and methods}

The material has been fixed in formalin, routinely processed and paraffin embedded. From the paraffin blocks, $2 \mu \mathrm{m}$ sections were prepared and stained by hematoxylin-eosin method. Immunohistochemistry was performed using a standard protocol. Briefly, the slides were dewaxed, rehydrated and incubated in a $3 \%$ peroxide solution for 8 minutes to block endogenous peroxidase ac- tivity. Antigen retrieval was carried out by water bath cooking in citrate buffer $(0.2 \%$ citric acid titrated to $\mathrm{pH}$ 6.0 with $2 \mathrm{~N} \mathrm{NaOH}$ or, for CD31 and VEGFR-3 staining, $0.037 \%$ EDTA in pH 8.0 instead of citric acid in $\mathrm{pH}$ 6.0) for 30 minutes. Primary antibodies are listed in Table I. The LabVision (Thermo Fisher Scientific, USA) detection system was used. 3,3'-diaminobenzidine (DAB) served as the chromogen. The slides were counterstained with Mayer's hematoxylin (Thermo Scientific, USA). Statistics were calculated with Statistica 10 software (StatSoft Inc., Tulsa, USA). Pearson's $\chi^{2}$ and Fisher exact test were used; the significance level was set at 0.05 .

\section{Results}

The group under study consisted of 6 cases; there were 3 males and 3 females. The age of the patients ranged from 16 to 82 (average 49). Lesions were located on the foot, trunk, lip, shin, mucosa of the nasal vestibulum and on the tongue.

Grossly, the lesions presented as reddish, soft nodules, measuring from $3 \mathrm{~mm}$ to $12 \mathrm{~mm}$ in diameter. Histologically, the superficial part of the lesions consisted of dilated vessels, some of them containing red blood 
Table I. Primary antibodies used in the study

\begin{tabular}{lccc}
\hline SPECIFICITY & ManufaCturer & ClONe/TyPE & Dilution \\
\hline CD31 & DAKO & JC70A & $1: 20$ \\
\hline CD34 & DAKO & QBEnd 10 & $1: 50$ \\
\hline Ki-67 & DAKO & MIB-1 & $1: 100$ \\
\hline D2-40 & COVANCE & D2-40 & RTU \\
\hline VIII-factor & DAKO & Fg/86 & $1: 50$ \\
\hline CD105 & Novocastra & 4G11 & $1: 50$ \\
\hline VEGFR-3 & Novocastra & KLT9 & $1: 50$ \\
\hline BCL-2 & Novocastra & bcl-2/100/D5 & $1: 50$ \\
\hline GLUT-1 & Cell Marque & policlonal & $1: 50$ \\
\hline RTU-ready to use & & &
\end{tabular}

cells (Fig. 1), and, in the deeper portion, also smaller non-arborizing vessels forming slit-like spaces, some of them dissecting collagen fibers. The endothelial cells in the superficial portion of the lesion were plump and exophytic, showing 'hobnail' morphology but the cells lining deeper spaces tended to be flat and inconspicuous (Fig. 2). Immunohistochemically, the endothelia in all 6 cases were positive for the usual endothelial markers, namely, CD31 (Fig. 3), CD34 and vWF. In all cases single endothelial cells expressed the Ki67 proliferation antigen. In 4 cases, the endothelia were also positive for CD105 and in 4 cases, some of the smaller vessels within the lesions showed positive reaction with the D2-40 antibody (Fig. 4). In 4 cases, single cells only were positive for BCL-2 staining. Results of the CD105, D2-40 and bcl-2 staining were not correlated. In all 6 cases, endothelia were negative for VEGFR-3 and GLUT-1 staining.

These results speak against lymphatic origin of $\mathrm{HH}$.

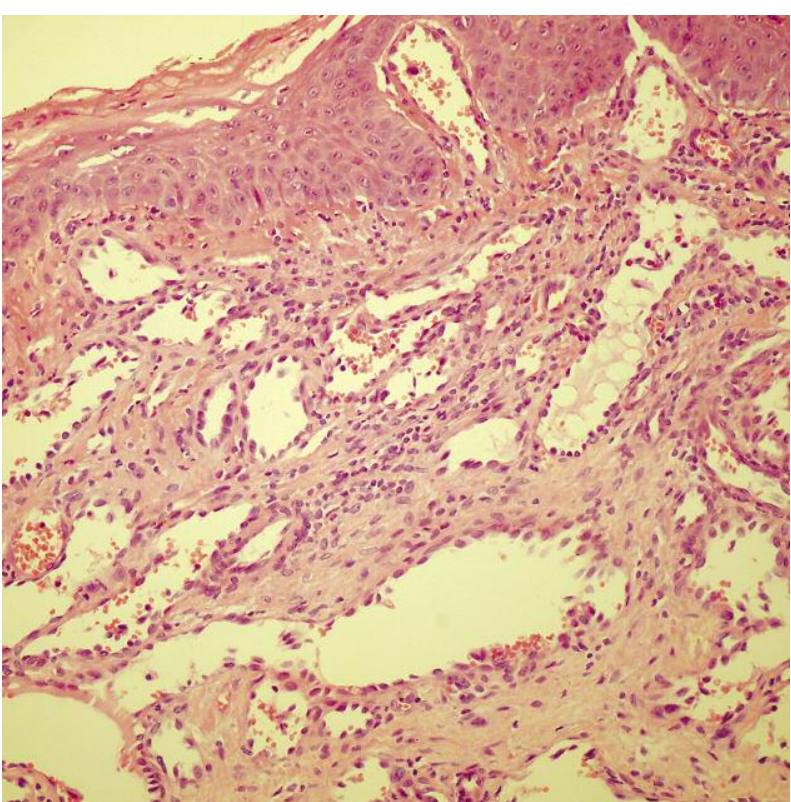

Fig. 1. Dilated superficial vessels of $\mathrm{HH}$, with red blood cells in their lumens. HE, magnification $200 \times$

\section{Discussion}

Hobnail hemangioma is a rare yet well-defined entity, its histogenesis however remains controversial. First described as targetoid hemosiderotic hemangioma [1], it may be seen in any age, but most patients are in their 20 s or 30s [5]. It may be located on skin of the trunk or extremities, and less frequently - the head and neck; mucosal location is even rarer [6].

The exact origin and etiology of $\mathrm{HH}$ remain controversial. Immunohistochemically, the endothelial cells are positive for such markers as CD31, CD34 and vWF; some studies however emphasized positivity for such lymphatic endothelial markers as podoplanin or VEFGR-3; there are even authors reporting $\mathrm{HH}$ to be D2-40 positive, CD34 negative. In contrast to other vascular lesions, $\mathrm{HH}$ lacks actin-containing pericytes [7-9]. These features would suggest lymphatic origin [5]. The WT1 gene and its protein product are well known to be involved in urogenital development and pediatric renal cancer; it has however other functions, including participation in angiogenesis. In fact, the expression of WT1 is seen in bone marrowderived stem cells developing into endothelial cells. IHC stain with WT1 has shown to be useful for the discrimination between vascular neoplasms and malformations, the former showing cytoplasmic positive stain for WT1, whereas the latter - negative. Trindade et al. [10] have analysed 52 cases of $\mathrm{HH}$, and reported them to be negative for WT1, while 8 cases were diffusely and 8 focally positive for D2-40. This was interpreted as characteristic of a lymphatic malformation rather than a lymphatic neoplasm. Al Dhaybi et al. [11] reported all 12 pediatric $\mathrm{HH}$ cases to be positive

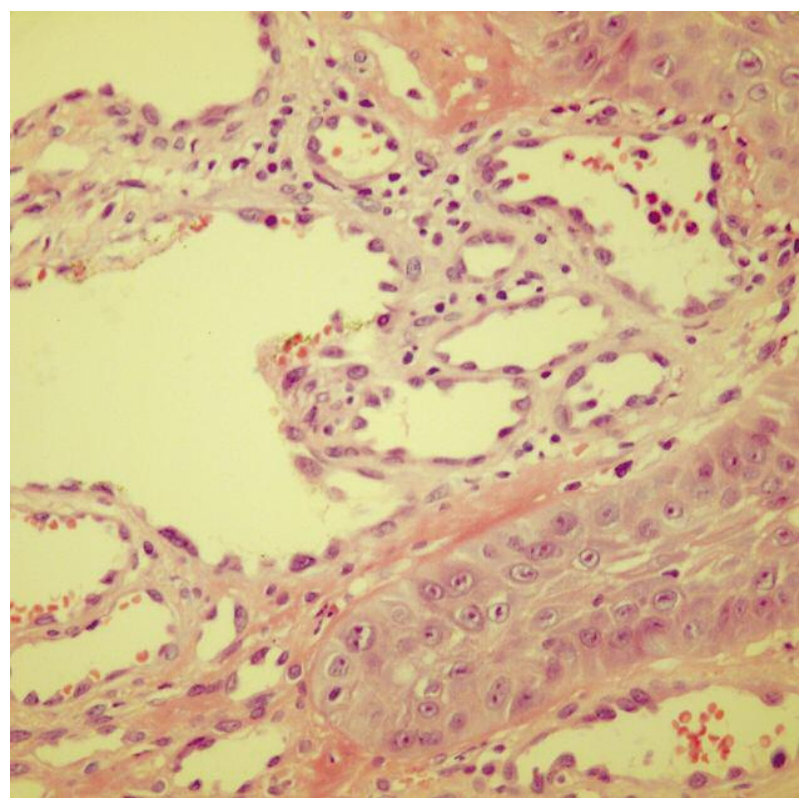

Fig. 2. Characteristic "Hobnail" morphology of the $\mathrm{HH}$ endothelial cells. HE, magnification $400 \times$ 


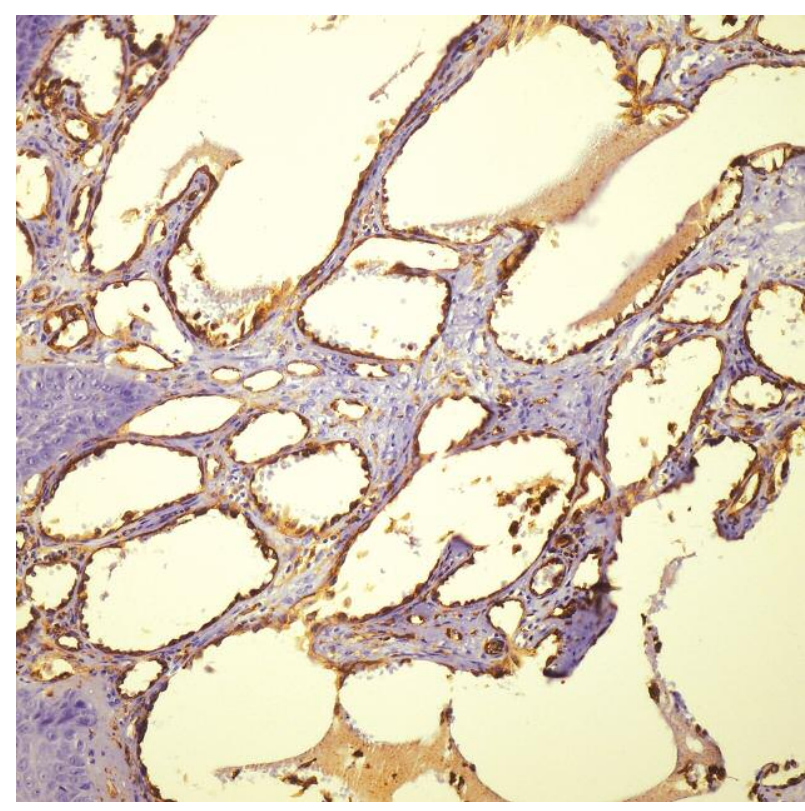

Fig. 3. Positive stain for $\mathrm{CD} 31$ in $\mathrm{HH}$ endothelial cells. Immunohistochemistry, magnification $200 \times$

for D2-40 and negative for GLUT-1. WT-1 was only focally positive in 2 cases. The authors suggested the name of "targetoid hemosiderotic lymphatic malformation" as more appropriate. On the other hand, in a review of Korean literature, Yoon et al. [12] reported all cases to be positive for blood vessel markers, but the stain for D2-40 was only focal. Mentzel et al. [8] reported all 28 out of $28 \mathrm{HH}$ cases to be positive for CD31 and negative for D2-40. Three cases were positive for CD34. Similarly, in Guillou et al. series [13], all 8 cases were positive for CD31 and negative for D2-40. These results are in concordance with the view that $\mathrm{HH}$ is a vascular and not lymphatic lesion. The results by Franke et al. [7] may suggest that $\mathrm{HH}$ is a biphasic lesion, carrying both lymphatic and vascular features, as all 10 cases were positive for CD31 and D2-40 and 6 were positive for CD34. The results in our series are similar.

In Mentzel et al. study [8], all 28 analyzed cases were CD 31 positive, but only 3 were fully CD 34 positive. 4 out of 8 cases were VEGFR-3 positive and 7 out of 27 cases had actin-positive pericytes. Mentzel suggests that $\mathrm{HH}$ represents a type of lymphangioma with characteristic cytomorphology. Guillou et al. [13] analysed 15 cases of cutaneous HH. Immunohistochemistry performed in 8 cases showed variable reactivity of endothelial cells with CD31, CD34, factor VIII-related antigen, and Ulex europaeus agglutinin-1. In all cases, smooth muscle actin-positive pericytes were observed focally around some of the abnormal vascular spaces. Also these results might suggest that $\mathrm{HH}$ is a specific type of lymphangioma.

HHV-8 immunohistochemistry in $\mathrm{HH}$ is negative [10], and Gutzmer et al. reported no HHV-8 DNA by PCR reaction [14]. Absence of HHV-8 in HH may be

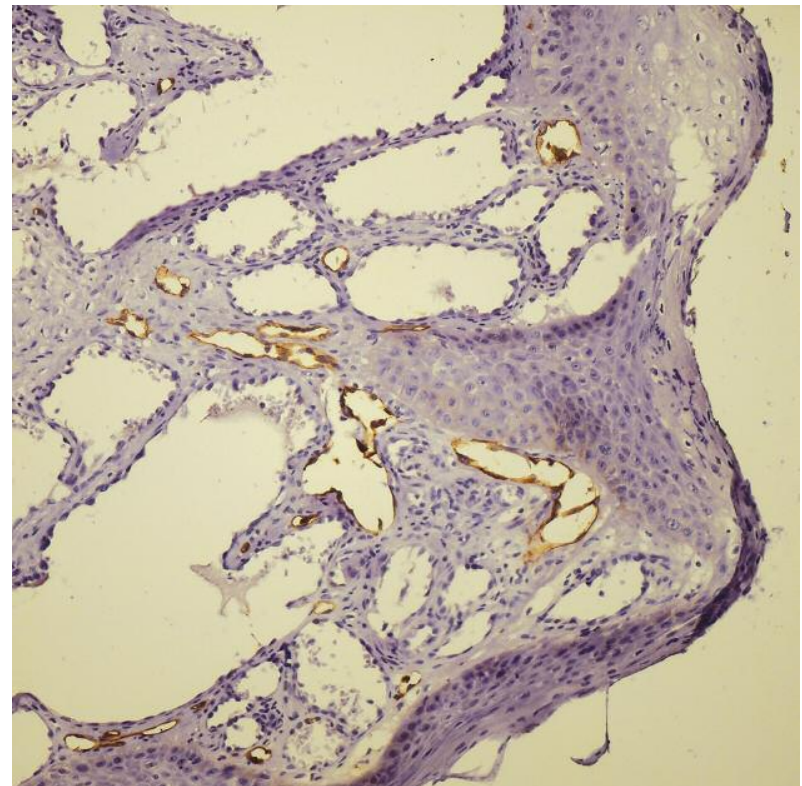

Fig. 4. Positive stain for D2-40 in some $\mathrm{HH}$ vessels. Immunohistochemistry, magnification $200 \times$

of practical importance for the differential diagnosis with Kaposi sarcoma.

It has also been proposed that $\mathrm{HH}$ might result from a simple physical injury or inflammation, like more common lobulated capillary hemangioma [5, 1517]. Others stress the role of the microshunts connecting lymphatic vessels and adjacent blood vessels [5]. Although stains for estrogen, progesterone and androgen receptor were reported as negative $[15,16]$, menstrual cycle and pregnancy dependence has been reported in female patients $[5,15]$.

In conclusion, the exact nature of hobnail hemangioma remains elusive. However, the recent results suggest that $\mathrm{HH}$ can be a trauma-related/post-traumatic vascular malformation.

\section{References}

1. Santa Cruz DJ, Aronberg J. Targetoid hemosiderotic hemangioma. J Am Acad Dermatol 1988; 19: 550-558.

2. Hejnold M, Dyduch G, Mojsa I, et al. Hobnail hemangioma. Pol J Pathol 2012; 64: 138-141.

3. Dyduch G, Okoń K. Proliferation and apoptosis within the oral mucosa "hemangiomas". Pol J Pathol 2000; 51: 93-96.

4. Dyduch G, Okoń K, Mierzyński W. Benign vascular proliferations - an immunohistochemical and comparative study. Pol J Pathol 2004; 55: 59-64.

5. Ibrahim M, Shwayder T. Hobnail hemangioma in a nine-yearold boy: a rare case presented with dermoscopy. Dermatol Online J 2010; 16: 7.

6. Rashmi MS, Alka KD, Seema C. Oral hobnail hemangioma a case report. Quintessence Int 2008; 39: 507-510.

7. Franke FE, Steger K, Marks A, et al. Hobnail hemangiomas (targetoid hemosiderotic hemangiomas) are true lymphangiomas. J Cutan Pathol 2004; 31: 362-367.

8. Mentzel T, Partanen TA, Kutzner H. Hobnail hemangioma ("targetoid hemosiderotic hemangioma"): clinicopathologic and immunohistochemical analysis of 62 cases. J Cutan Pathol 1999; 26: 279-286. 
9. Pabuccuoğlu U, Ozkara E, Lebe B. Hobnail haemangioma occurring on the nasal dorsum. Br J Dermatol 2002; 146: 162 164.

10. Trindade F, Kutzner H, Tellechea Ó, et al. Hobnail hemangioma reclassified as superficial lymphatic malformation: a study of 52 cases. J Am Acad Dermatol 2012; 66: 112-115.

11. Al Dhaybi R, Lam C, Hatami A, et al. Targetoid hemosiderotic hemangiomas (hobnail hemangiomas) are vascular lymphatic malformations: a study of 12 pediatric cases. J Am Acad Dermatol 2012; 66: 116-120.

12. Yoon SY, Kwon HH, Jeon HC, et al. Congenital and multiple hobnail hemangiomas. Ann Dermatol 2011; 23: 539-543.

13. Guillou L, Calonje E, Speight P, et al. Hobnail hemangioma: a pseudomalignant vascular lesion with a reappraisal of targetoid hemosiderotic hemangioma. Am J Surg Pathol 1999; 23: 97-105.

14. Gutzmer R, Kaspari M, Herbst RA, et al. Absence of HHV-8 DNA in hobnail hemangiomas. J Cutan Pathol 2002; 29: 154158.

15. Hong WK, Song HJ, Lee HS, et al. Hobnail haemangioma associated with a secondary sexual characteristic. J Eur Acad Dermatol Venereol 2009; 23: 465-466.

16. Ortiz-Rey JA, González-Ruiz A, San Miguel P, et al. Hobnail haemangioma associated with the menstrual cycle. J Eur Acad Dermatol Venereol 2005; 19: 367-369.

17. Sahin MT, Demir MA, Gunduz K, et al. Targetoid haemosiderotic haemangioma: dermoscopic monitoring of three cases and review of the literature. Clin Exp Dermatol 2005; 30: 672-676.

\section{Address for correspondence}

\section{Maria Hejnold}

Department of Pathomorphology

Collegium Medicum

Jagiellonian University

ul. Grzegórzecka 16

31-531 Kraków

e-mail: wirtualka10@wp.pl 